\title{
Correction: Evaluation of ERK as a therapeutic target in acute myelogenous leukemia
}

\author{
Ellen Weisberg $(\mathbb{D} \cdot$ Chengcheng Meng • Abigail Case • Martin Sattler · Hong L. Tiv • Prafulla C. Gokhale • \\ Sara Buhrlage · Jinhua Wang · Nathanael Gray (10) Richard Stone - Suiyang Liu - Shripad V. Bhagwat - Ramon V. Tiu • \\ Sophia Adamia $\cdot$ James D. Griffin
}

Published online: 6 March 2020

(c) Springer Nature Limited 2020

\section{Correction to: Leukemia}

https://doi.org/10.1038/s41375-019-0552-3
Following the publication of this article, the authors noted minor errors in Supplementary Fig. 5. The correct version of this figure is included below along with the accompanying figure legend.
A

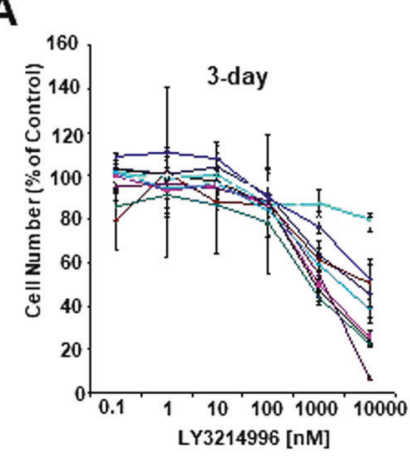

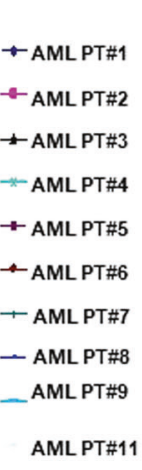

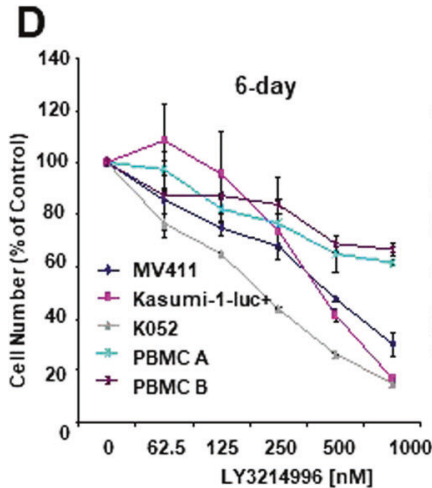

B

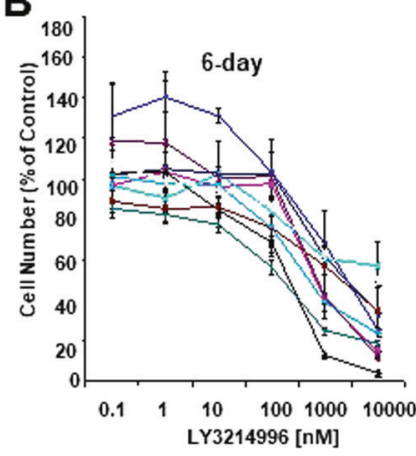

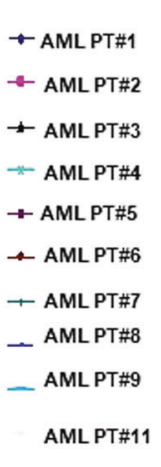

$\mathbf{F}$
C

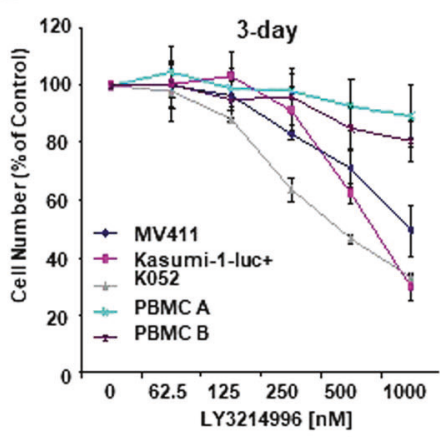

E

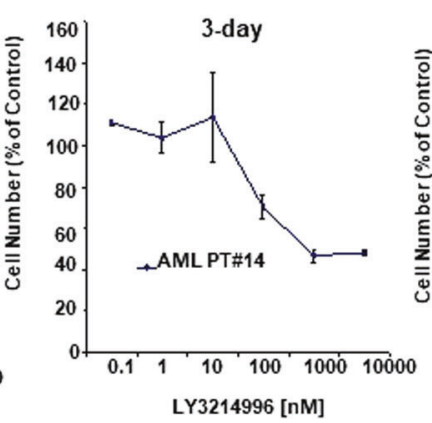

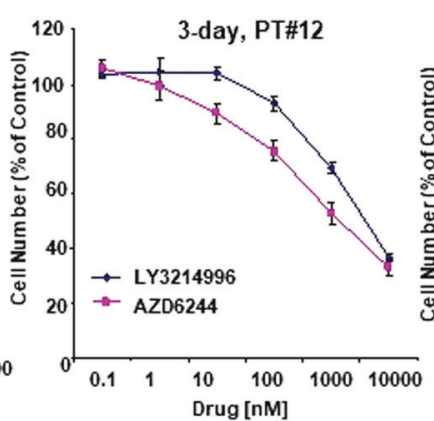

G

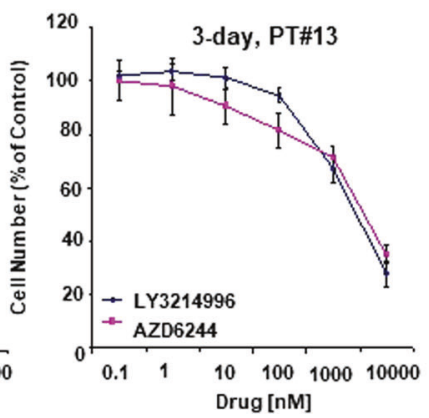

Supplementary Fig. 5 LY3214996 potently inhibits proliferation of human primary patient AML cells in a concentration-dependent manner. (A, B) Proliferation studies; 3-day LY3214996 treatment (A) or 6-day treatment (B) of primary AML patient samples. (C, D) Proliferation studies; 3-day LY3214996 treatment (C) or 6-day treatment (D) of normal PBMCs versus human AML cells. (E) LY3214996 treatment of AML patient sample 14 for 3 days. (F-G) Proliferation studies; 3-day LY3214996 or AZD6244 treatment of AML patient samples $12(\mathrm{~F})$ and $13(\mathrm{G})$. Patient and primagraft information for all samples shown in this manuscript is provided in Supplementary Tables 1 and 2. 